\title{
Functional Pain Syndromes: Presentation and Pathophysiology
}

\author{
Emeran A. Mayer, M. Catherine Bushnell (eds). IASP Press, Seattle, WA, 2009, \\ 580 pp. ISBN 978-0-931092-75-6
}

\author{
Tania Di Renna, MD
}

Published online: 6 August 2009

(C) Canadian Anesthesiologists' Society 2009

In their foreword to this book, the editors describe the pathophysiology of "functional" pain syndromes as remaining poorly understood. They have undertaken the overwhelming task of clarifying the possible pathophysiology of diverse pain syndromes that have remained a mystery over the decades. Their focus is on the ever elusive functional pain syndromes or disorders that have no identifiable physiologic cause, such as irritable bowel syndrome, vulvodynia, fibromyalgia, interstitial cystitis, and temporomandibular joint disorder. The mechanistic pathways of these syndromes remain nebulous and often result in patients being mismanaged and misdiagnosed.

The book is a collaboration of an international panel of expert scientists, researchers, and clinicians in pain management. The experts describe the essential features of common functional syndromes and attempt to bridge the gap in understanding between the basic science and the clinical management of each disorder.

The first three sections of this seven part text focus on the somatic, visceral, and common comorbid syndromes, respectively. The following two sections describe neurobiological and genetic mechanisms as possible contributors to chronic pain syndromes, while the final chapters deal with treatment strategies.

The initial seven chapters present a general overview and description of the possible pathophysiology of various somatic and visceral pain syndromes. The genetic, behavioural, and comorbid conditions are described for each disorder. The information is described clearly and referenced appropriately. In the chapters following, the roles in functional pain syndromes assumed by psychiatric factors, immunity, and the autonomic nervous system are astutely described. Illustrations, tables, and graphs are suitably integrated into the text. The remaining chapters, which I found most useful, include evidence-based discussions on pharmacologic and non-pharmacologic treatment strategies.

Overall, this text provides current and in-depth information on functional chronic pain syndromes. I would recommend it as a reference text for any fellow or physician specializing in chronic pain management and research.

T. Di Renna, MD $(\bowtie)$

University of Ottawa, Ottawa, Canada

e-mail: tdirenna@hotmail.com 